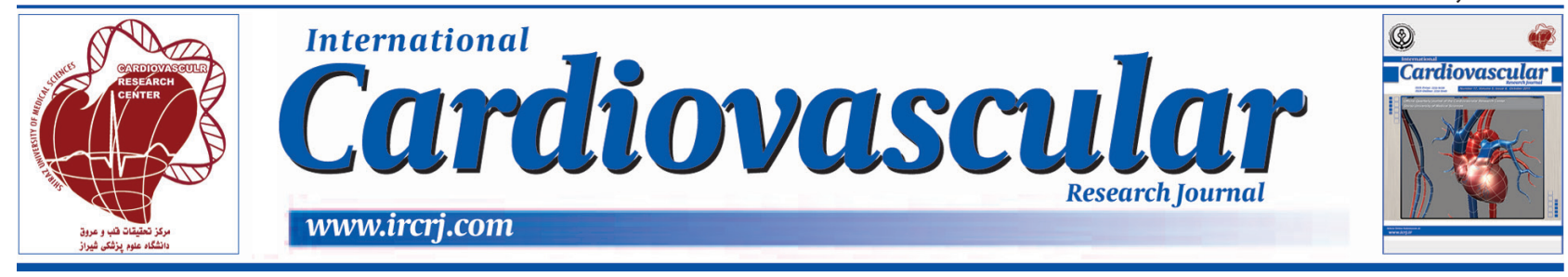

\title{
Upregulation of Ryanodine Receptor Calcium Channels (RyR2) in Rats with Induced Diabetes after 4 Weeks of High Intensity Interval Training
}

\author{
Mohammad Ali Babaee Bigi, ${ }^{1}$ Hossein Faramarzi, ${ }^{2}$ Abbas Ali Gaeini, ${ }^{3}$ Ali Asghar Ravasi, ${ }^{3}$ \\ Mohammad Reza Izadi, ${ }^{3,}{ }^{*}$ Maryam Delfan, ${ }^{3}$ and Esmaeil Izadi ${ }^{4}$
}

${ }^{1}$ Cardiovascular Research Center, Shiraz University of Medical Sciences, Shiraz, IR Iran

${ }^{2}$ Larestan School of Medical Science, Larestan, IR Iran

${ }^{3}$ Faculty of Sport Science, Tehran University, Tehran, IR Iran

${ }^{4}$ Shiraz School of Pharmacy, Shiraz University of Medical Sciences, Shiraz, IR Iran

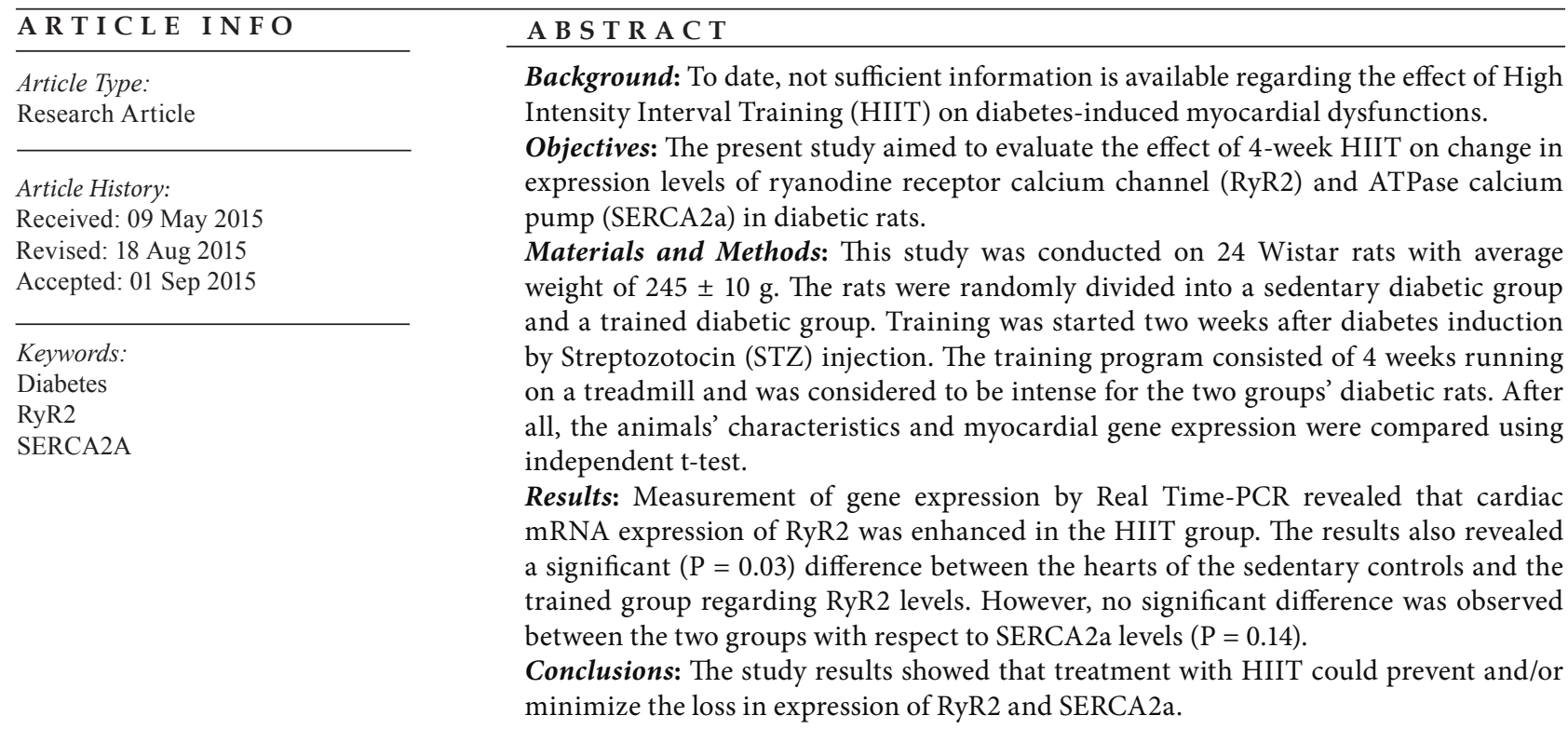

Implication for health policy/practice/research/medical education:

The present study aimed to evaluate the relationship between change in RyR2 gene expression level and SERCA2a after 4 weeks of high intensity interval training in rats with induced diabetes. The results provided a possible explanation for the decrease in diabetic cardiomyopathy seen among the diabetics involved in high intensity interval training.

\section{Background}

Studies have shown that coronary atherosclerosis and cardiomyopathy are caused by diabetes-based unnatural metabolism (1). Diabetic cardiomyopathy has been diagnosed in clinical and animal diabetic models without any symptom of vascular damage (2). At molecular level, these changes are probably derived from changes in

*Corresponding author: Mohammad Reza Izadi, North Kargar Avenue, Faculty of Sport Science, Tehran University, Tehran, Iran. Tel: +98-7136704338,

E-mail:izadi.mreza@gmail.com expression and/or activity of various proteins involved in maintaining or regulating intracellular calcium homeostasis (3, 4). Ryanodine receptors (RyR2) are among these groups of proteins. They include intracellular calcium-releasing channels in the sarcoplasmic reticulum membrane (5). Returning to diastolic mode for cardiac expansion in calcium cycle is done by calcium ATPase pump of sarcoplasmic reticulum (SERCA2a) (6). Cardiac expansion begins following the process of calcium delay. This event is controlled by calcium release and collection 
mechanism. SERCA pump pumps calcium from cytosol to sarco-endoplasmic reticulum in the process of calcium collection (7). Any change in the sensitivity of RyR2, SERCA2a, and calcium-based activities (derangement of two protein) in chronic diabetes is partly responsible for reduction in myocardial speed and contraction (8). In addition to decrease in gene expression and proteins regulating myocardial calcium cycles, integration and intact activity of ryanodine was decreased in rats with Streptozotocin (STZ)-induced diabetes. In these rats, a significant decrease was reported in expression of genes and regulator proteins, such as phospholamban, RyR2, and SERCA2a (3).

Training protocols are one of the most effective methods to reduce the progress of cardiomyopathy, cardiovascular disorders, and death due to diabetes. Studies on rates with type 1 diabetes have shown that most training protocols affected factors, such as ejection fraction and stroke volume, in rats with diabetes-induced cardiac disorders $(9,10)$. High Intensity Interval Training (HIIT) is more effective and more useful than intermediate intensity continuous training in increasing aerobic capacity and cardiovascular functions in healthy individuals and patients with cardiovascular diseases (11-13). Also, in comparison to traditional exercises with low to intermediate intensity, these types of training were more effective in cardiac revival, controlling blood sugar and most other clinical symptoms of diabetes, and the risk factors affecting cardiac patients in the long run $(14,15)$.

In general, RyR2 receptors become more sensitive to calcium function due to diabetes and more calcium is required in order to inactivate the aforementioned channels. Training protocols slow down the changes in the amounts of calcium. However, a limited number of studies have been conducted on the underlying mechanisms affecting RyR2 inactivity or sensitivity to calcium function (8). Moreover, covert mechanisms of the effect of exercise on diabetic heart are not known yet. Furthermore, although it has been confirmed that myocardial adaptation with exercise depends on the intensity of training schedules in healthy animals, few comprehensive studies have examined the duration and intensity of exercises and their regulating effects on the function of diabetic heart (16).

\section{Objectives}

The present study aims to assess the mechanisms involved in damaging, modifying, and regulating gene expression of RyR2 and SERCA2a in diabetic hearts in response to these types of exercises.

\section{Materials and Methods}

\subsection{Experimental Models and Injection of Streptozotocin}

This study was performed on 24 Wistar rats with average weight of $245 \pm 10 \mathrm{~g}$. At first, $50 \mathrm{mg} / \mathrm{kg}$ body weight of STZ in buffer citrate solution $(\mathrm{pH}: 4.5)$ was injected intraperitoneally to the rats in a single dose. Four days after STZ injection, blood sugar level was measured by 0 1 glucometer (made in Japan) in order to confirm diabetes. Blood sugar level above $300 \mathrm{mg} / \mathrm{mL}$ was considered to be the indicator of diabetes. Two weeks after diabetes induction, the rats were randomly divided into two groups (sedentary control and HIIT) each containing 12 animals. At this stage, the rats got familiar with treadmill (four rats were selected as pilot protocols). Training protocol of the HIIT group consisted of 24 minutes of running on treadmill. However, the control group did not participate in any training schedules.

\subsection{Training Protocol}

Two weeks after diabetes induction by STZ injection, the rats started their training schedule as follows: five minutes warm-up with 30 - 40\% VO2max intensity, 3 minutes with $58-90 \%$ VO2max intensity, one minute recovery with 3 $35 \% \mathrm{VO} 2 \mathrm{max}$ intensity, and five minutes cool-down with $30-40 \%$ VO2max intensity. The HIIT group performed this protocol five days a week for four weeks.

\subsection{Real-Time-PCR Analysis}

Real-time PCR was used to quantitatively measure the messenger RNA (mRNA) levels of RyR2 and SERCA2a. In doing so, the cells' RNA was extracted and treated following DNAse I treatment. In this method, extra DNA was removed from the sample. Ultimately, cDNA was made and PCR-qRT reactions were done. The extent of expression of the intended mRNAs was measured according to 2- $\Delta \Delta \mathrm{ct}$.

\subsection{Statistical Analysis}

All the analyses were performed using the SPSS statistical software, version 16. The animals' characteristics and myocardial gene expression were compared using independent t-test. All the values were expressed as mean \pm SEM and $\mathrm{P}<0.05$ was considered to be statistically significant.

\section{Results}

The general characteristics of the rats at the time of sacrifice have been presented in Table 1. As expected, the level of blood sugar was higher in the sedentary diabetic rats than in the training group. Thus, training reduced the blood sugar levels. Besides, body weight reduced in the sedentary group, but slightly increased in the training group (Figures 1 and 2).

\section{Discussion}

Previous clinical studies demonstrated that exercise training slowed down and/or delayed the progression of myocardial contractility loss induced by both type 1 and type 2 diabetes. However, molecular mechanisms underlying this beneficial effect have remained incompletely characterized.

\begin{tabular}{lcc}
\hline \multicolumn{2}{l}{ Table 1. General Characteristics of the Animals Used in the Study $(\mathrm{P} \leq 0.05$ Indicated Significant Difference Between the two Groups.) } \\
\hline Parameter & \multicolumn{1}{c}{ Sedentary Control Group } & Training Group \\
\hline Weight $(\mathbf{g})$ & $238.17 \pm 17.9$ & $276.4 \pm 16.5$ \\
Blood sugar $(\mathbf{m g} / \mathbf{d m})$ & $577.6 \pm 11.5$ & $502.8 \pm 13.4$ \\
\hline
\end{tabular}


Figure 1. Measurement of RyR2 Gene Expression Fold Change by RT-PCR

\section{RyR2}

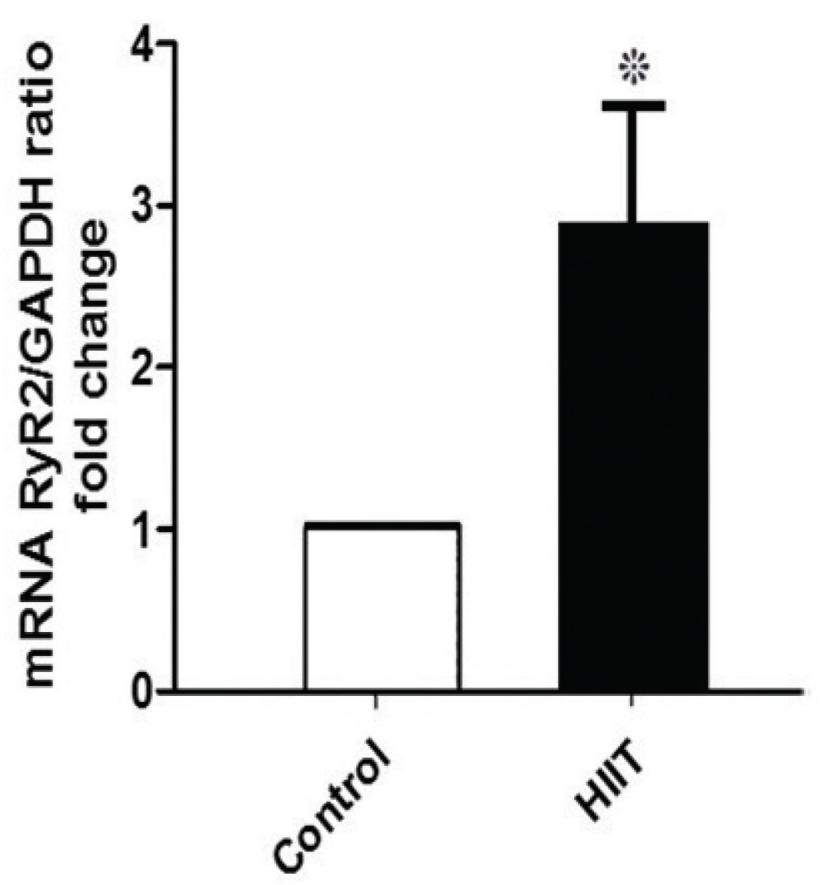

The effect of HIIT on the expression of RyR2 gene in the rats. Experiments on the control rats were performed in triplicate and PCR quantification was done by densitometry analysis.

${ }^{*} \mathrm{P} \leq 0.05$ indicated significant difference between the two groups.

In the present study, a multifaceted approach was used that revealed for the first time that HIIT during diabetes minimized irregularity of RyR2. The results showed that HIIT normalized or reduced down-regulation of RyR2 and dysfunction of both systolic and diastolic parameters in cardiomyocytes from diabetic cardiomyopathy hearts. Patients with chronic diabetes also showed severe systolic dysfunction, which was probably due to changes in the expression and function of numerous genes and proteins involved in regulating/maintaining intracellular calcium homeostasis (3).

In this study, we focused on the effects of HIIT on expression and function of two proteins, namely RyR2 and SERCA2a. The study findings demonstrated that the cardiac mRNA expression of RyR2 $(1.847 \pm 0.65, \mathrm{P}<$ $0.05)$ and SERCA2a $(1.206 \pm 0.11, \mathrm{P}<0.05)$ enhanced in the HIIT group. The results also revealed a significant $(\mathrm{P}=0.03)$ difference between the sedentary control and trained animals regarding RyR2 levels. However, no significant difference was found between the two groups concerning SERCA2a levels $(\mathrm{P}=0.14)$. Moreover, the increased expression of RyR2 was reversed (upregulated) in the diabetic rats after performance of HIIT. Similarly, previous studies demonstrated that different exercise training protocols led to an increase or a change in RyR2 expression at mRNA or protein levels in diabetic rats induced by STZ compared to the age-matched control rats. In the study conducted by Salem et al. (2013), daily 1-hour exercise training sessions were repeated 5 days a week for 2 - 3 months. Their program was focused on pyramidal continuous exercise training protocol. The results of that study indicated no significant difference between sedentary Goto-Kakizaki (GK) and sedentary control rats with respect to expression of mRNA encoding intracellular $\mathrm{Ca} 2+$ transport and RyR2 regulatory protein (17). Chun Hong Shao et al. (2009) also assessed total and phosphorylation forms of RyR2 antibody in rats after 4 weeks of exercise training by ascending continuous protocol. They found that the phosphorylation form of RyR2 was increased in the exercise training group, but the total form of RyR2 remained unchanged (8).

Alterations in RyR2 and phospholamban could regulate SERCA2a function in the heart, which controls Ca2+ loading and magnitude of $\mathrm{Ca} 2+$ transients $(8,18)$. SERCA2a is the main contributor to removal of cytosolic $\mathrm{Ca} 2+$ during diastole. Similar to the previous studies, our study results showed that exercise training restored the functions of SERCA2a to levels comparable to those of the sedentary control group. This suggested that exercise training normalized diastolic function in the trained group by shifting the control of diastolic $\mathrm{Ca} 2+$ to SERCA2a and, consequently, increased the rate of $\mathrm{Ca} 2+$ removal. This also increased the SERCA2a load, which might have contributed to improvement of systolic function.

Several researches have reported that the effects of exercise on the cardiovascular system depend on the intensity or amount of exercise (19-21). The intensity and duration of the exercise used in our study were comparable with those in the majority of the previous studies conducted on diabetic cardiomyopathy. Additionally, several studies 


\section{SERCA2a}

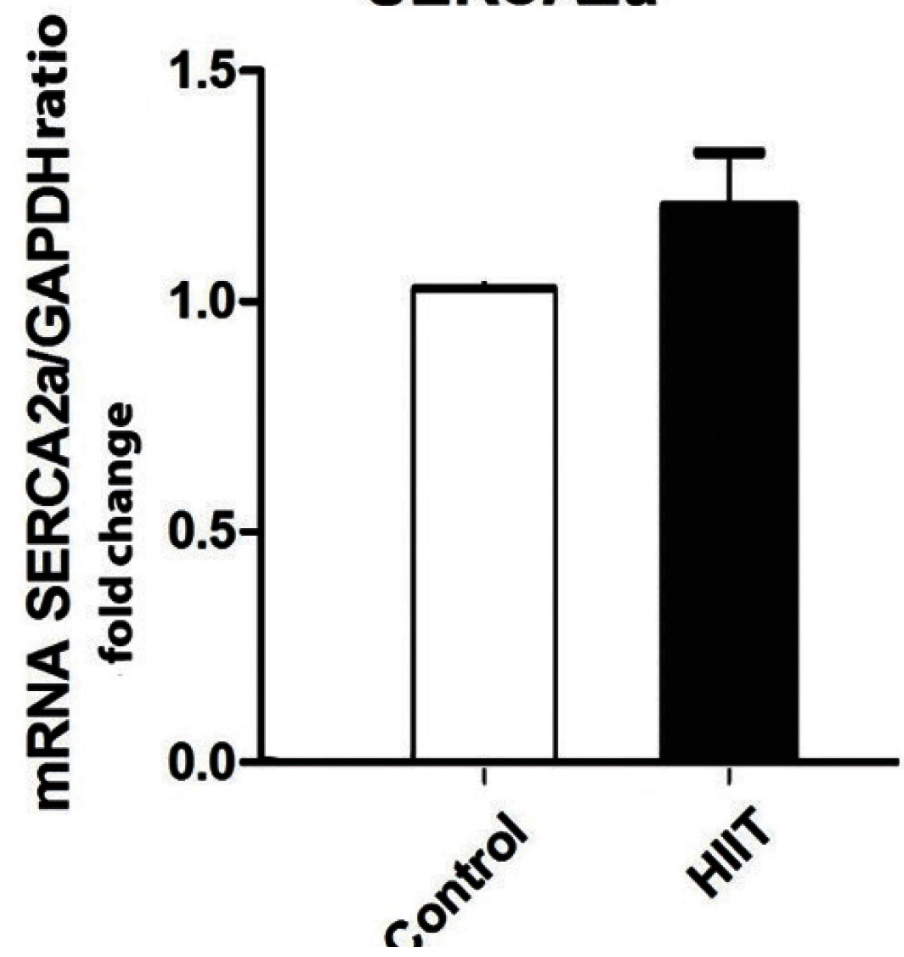

The effect of HIIT on the expression of SERCA2a gene in the rats. Experiments on the control rats were performed in triplicate and PCR quantification was done by densitometry analysis.

${ }^{*} \mathrm{P} \leq 0.05$ indicated significant difference between the two groups.

have demonstrated the beneficial effects of exercise on myocardial muscle glucose homeostasis (9). High intensity and short duration of exercise might have played a role in our study. In this study, exercise training reduced blood sugar level to a small extent in the HIIT group compared to the age-matched controls.

In conclusion, the findings of the current study showed that HIIT could prevent and/or minimize the loss in expression of RyR2 and SERCA2a. Thus, high intensity and short duration of exercise might have played a role in prevention of decreased gene expression in diabetic rats' hearts in our study.

\section{Acknowledgements}

There is no acknowledgement.

\section{Authors' Contribution}

Mohammad Ali Babaei Bigi and Hossein Faramarzi funded this project and contributed to the research design. Mohammadreza Izadi was the corresponding author. Abbas Ali Gaeini, Ali Asghar Ravasi, and Delfan contributed to the research design and laboratory works. Esmaeil Izadi participated in pharmacological guidance.

\section{Financial disclosure}

There is no financial disclosure.

\section{Funding/Support}

This project was funded by Larestan University of Medical
Sciences.

\section{References}

1. Grundy SM, Benjamin IJ, Burke GL, Chait A, Eckel RH, Howard $\mathrm{BV}$, et al. Diabetes and cardiovascular disease: a statement for healthcare professionals from the American Heart Association. Circulation. 1999;100(10):1134-46.

2. Rodrigues B, Cam MC, McNeill JH. Myocardial substrate metabolism: implications for diabetic cardiomyopathy. J Mol Cell Cardiol. 1995;27(1):169-79.

3. Bidasee KR, Nallani K, Yu Y, Cocklin RR, Zhang Y, Wang M, et al. Chronic diabetes increases advanced glycation end products on cardiac ryanodine receptors/calcium-release channels. Diabetes. 2003;52(7):1825-36.

4. Shizukuda Y, Reyland ME, Buttrick PM. Protein kinase C-delta modulates apoptosis induced by hyperglycemia in adult ventricular myocytes. Am J Physiol Heart Circ Physiol. 2002;282(5):H1625-34.

5. Dincer UD. Cardiac ryanodine receptor in metabolic syndrome: is JTV519 (K201) future therapy? Diabetes Metab Syndr Obes. 2012;5:89-99.

6. Duan J, Zhang HY, Adkins SD, Ren BH, Norby FL, Zhang X, et $a l$. Impaired cardiac function and IGF-I response in myocytes from calmodulin-diabetic mice: role of Akt and RhoA. Am J Physiol Endocrinol Metab. 2003;284(2):E366-76.

7. Sheikh AQ, Hurley JR, Huang W, Taghian T, Kogan A, Cho H, et al. Diabetes alters intracellular calcium transients in cardiac endothelial cells. PLoS One. 2012;7(5):e36840.

8. Shao CH, Wehrens XH, Wyatt TA, Parbhu S, Rozanski GJ, Patel KP, et al. Exercise training during diabetes attenuates cardiac ryanodine receptor dysregulation. J Appl Physiol (1985). 2009;106(4):1280-92.

9. Howarth FC, Almugaddum FA, Qureshi MA, Ljubisavljevic M. Effects of varying intensity exercise on shortening and intracellular calcium in ventricular myocytes from streptozotocin (STZ)-induced diabetic rats. Mol Cell Biochem. 2008;317(1-2):161-7.

10. Pereira L, Matthes J, Schuster I, Valdivia HH, Herzig S, Richard S, et al. Mechanisms of [Ca2+]i transient decrease in cardiomyopathy 
of db/db type 2 diabetic mice. Diabetes. 2006;55(3):608-15.

11. Guimaraes GV, Ciolac EG, Carvalho VO, D'Avila VM, Bortolotto LA, Bocchi EA. Effects of continuous vs. interval exercise training on blood pressure and arterial stiffness in treated hypertension. Hypertens Res. 2010;33(6):627-32.

12. Haram PM, Kemi OJ, Lee SJ, Bendheim MO, Al-Share QY, Waldum $\mathrm{HL}$, et al. Aerobic interval training vs. continuous moderate exercise in the metabolic syndrome of rats artificially selected for low aerobic capacity. Cardiovasc Res. 2009;81(4):723-32.

13. Wisloff U, Stoylen A, Loennechen JP, Bruvold M, Rognmo O, Haram $\mathrm{PM}$, et al. Superior cardiovascular effect of aerobic interval training versus moderate continuous training in heart failure patients: a randomized study. Circulation. 2007;115(24):3086-94.

14. Meyer P, Normandin E, Gayda M, Billon G, Guiraud T, Bosquet L, et al. High-intensity interval exercise in chronic heart failure: protocol optimization. J Card Fail. 2012;18(2):126-33.

15. Stolen TO, Hoydal MA, Kemi OJ, Catalucci D, Ceci M, Aasum E, et al. Interval training normalizes cardiomyocyte function, diastolic $\mathrm{Ca} 2+$ control, and SR Ca2+ release synchronicity in a mouse model of diabetic cardiomyopathy. Circ Res. 2009;105(6):527-36.

16. Le Douairon Lahaye S, Rebillard A, Zguira MS, Malarde L, Saiag B, Gratas-Delamarche A, et al. Effects of exercise training combined with insulin treatment on cardiac NOS1 signaling pathways in type 1 diabetic rats. Mol Cell Biochem. 2011;347(1-2):53-62.

17. Salem KA, Adrian TE, Qureshi MA, Parekh K, Oz M, Howarth FC. Shortening and intracellular $\mathrm{Ca} 2+$ in ventricular myocytes and expression of genes encoding cardiac muscle proteins in early onset type 2 diabetic Goto-Kakizaki rats. Exp Physiol. 2012;97(12):1281-91.

18. Wang M, Zhang WB, Zhu JH, Fu GS, Zhou BQ. Breviscapine ameliorates cardiac dysfunction and regulates the myocardial $\mathrm{Ca}(2+)$-cycling proteins in streptozotocin-induced diabetic rats. Acta Diabetol. 2010;47 Suppl 1:209-18.

19. Amatyakul S, Chakraphan D, Chotipaibulpan S, Patumraj S. Role of exercise training on pulpal blood flow in diabetic rats. Clin Hemorheol Microcirc. 2006;34(1-2):295-301.

20. Harthmann AD, De Angelis K, Costa LP, Senador D, Schaan $\mathrm{BD}$, Krieger EM, et al. Exercise training improves arterial baroand chemoreflex in control and diabetic rats. Auton Neurosci. 2007;133(2):115-20.

21. Loganathan R, Bilgen M, Al-Hafez B, Zhero SV, Alenezy MD, Smirnova IV. Exercise training improves cardiac performance in diabetes: in vivo demonstration with quantitative cine-MRI analyses. J Appl Physiol (1985). 2007;102(2):665-72. 